\title{
Introduction to the Seniors' Use of Digital Resources Minitrack
}

\author{
Heiko Gewald \\ Neu-Ulm University \\ heiko.gewald@hs-neu-ulm.de
}

\author{
Doug Vogel \\ Harbin Institute of Technology \\ isdoug@hit.edu.cn
}

\author{
Karoly Bozan \\ Duquesne University \\ bozank@duq.edu
}

The United Nations define ageing societies all over the world to be one of the grand challenges of humankind over the next few decades. By 2050, more than 2.1 billion human beings are predicted to be older than 60 years of age [1].

Information technology and information systems are widely expected to be able to help ease the challenges the ageing population puts on health systems worldwide by enabling senior citizens to live a longer self-sustaining life.

However, although the percentage of older people using digital resources continues to rise [2], research still has no thorough understanding about the specific wants and needs of this user group. This refers not only to declining cognitive and motoric abilities, but also to routines and habits of these users.

Going forward, IS research needs to provide answers to the resulting questions. Amongst those are for example:

1. Every year, millions of dollars are invested in developing digital resources for the ageing generation. However, most of these innovations do not make a lasting contribution to the well-being of the target population. A famous example is the so called "senior phone", i.e. cellphones specifically developed for elderly people. Although invented with the target group in mind, emerged as a best seller. Seniors simply do not want to use them. This underlines the necessity to provide development frameworks which incorporate the intended users and their specific living conditions and habits better than before.

2. Our conceptualization of "age" needs to be revisited. Physical age does not seem to serve as an adequate control variable anymore. Is 60 really the new 40 ? Is the aging population actually mentally (and physically?) younger than previous generations? The notion of cognitive/ perceived age becomes more important in research on the specifics of senior citizens. However, currently used measurements deserve to be re-visited to prove that they actually measure what they are supposed to measure, even though the seniors today seem to feel younger than ever before.
The papers in this minitrack address the grand challenge of ageing populations.

Bozan and Berger present their work "Revisiting the Technology Challenges and Proposing Enhancements in Ambient Assisted Living for the Elderly". They provide a review of the technological challenges that were identified as inhibiting factors to ambient-assisted living (AAL), which supports the elderly to decrease their dependency on formal or informal caregivers. They found that caregivers are predominantly untrained family members who could very much benefit from being aware of AAL technologies. Thus, they call for a new generation of ALL technology and emphasis on caretaker adoption. This approach may significantly reduce the serious consequences that result from delayed medical help due to medically untrained caretakers.

Bäccman and Bergkvist present their work "Welfare Technology and User Experience: a Case Study of Elderly Clients' Expectations on and First Impressions of a Robotic Shower". They analyze the adoption of AAL technologies, in this case a robotic shower. Their findings show that the acceptance of such technologies strongly depends on the specific context and cannot be viewed from one perspective for all technologies.

Hayes, Introna and Smith present their work on "Revealing and Designing Ensembles of Practice: Older Adults, Technology, and Loneliness \& Social Isolation in Rural Settings". They address an important and still under-researched area, namely how ICT plays a role in addressing loneliness and social isolation for older adults in rural areas. Their findings underline how important it is to include seniors and their specific habits in the actual conceptualization of digital resources. Thus, adopting a practice lens proves to be especially important for successful development.

We are looking forward to further researchers building on the knowledge presented in these papers and presenting their own research dealing with the issue of ageing populations.

\section{References}

[1] United Nations (2017), World Population Prospects - The 2017 Revision

[2] Pew Research Center (2017), Tech Adoption Climbs Among Older Adults 